\title{
BREAST-CONSERVING SURGERY AFTER NEOADJUVANT CHEMOTHERAPY IN PATIENTS WITH STAGE II AND III TREATED IN THE PUBLIC HEALTH SYSTEM
}

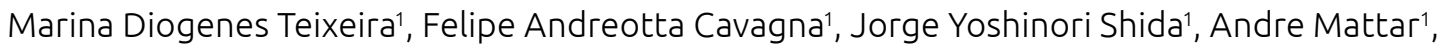
Luiz Henrique Gebrim

${ }^{1}$ Hospital Perola Byington - São Paulo (SP), Brazil.

Introduction: Over the last few decades, neoadjuvant chemotherapy (NCT) has played an increasing role in the management of breast cancer. It allows for an evaluation of the tumour reponse in vivo and may afford a de-escalation of surgeries. Despite advances in neoadjuvant treatment, evidence shows that rates of breast-conserving surgeries (BCT) after NCT are low (51\%-68\%) even when a patient is eligible for a less agressive surgery. Objectives: To analyze the surgical treatment performed in patients who underwent NCT in clinical stages (CS) II or III within a public health service in Brazil. Methods: A cross-sectional study was conducted with statistical analysis of the database of a public hospital in São Paulo with 11,073 patients treated from January 2009 to December 2020. Results: A total of 11,073 patients with breast cancer were treated in this period and 9,526 surgeries were performed, from which 4,613 (48.4\%) were BCS and 4,913 (51.6\%) were mastectomies. Among these procedures, 2,231 patients underwent NCT before surgical treatment, 275 (12\%) were submitted to BCS and 1,956 (88\%) to mastectomy. When compared by clinical stages: 641 were in CS II, BCS was performed in $143(22.3 \%)$ and mastectomy in 498 (77.7\%), and 1,590 were in CS III, from which 132 (8.3\%) were submitted to BCS and 1,458 (91.7\%) to mastectomy after neoadjuvant treatment. Conclusions: We observed that the rate of BCS after NCT in patients in CS II or III in our service was even lower than that found in the international literature. Some of the factors that may have influenced this result are: patient preference, anatomical extension of the tumor with skin ulceration (T4b), physician insecurity in performing a less aggressive treatment or difficulty accessing radiotherapy for patients from distant cities. This suggests that the potential surgical benefits of NCT are not being fully understood or explored. There is a need to resolve the uncertainties that are are holding back surgical teams from adopting more conservative surgeries. 